Bull. Korean Math. Soc. 48 (2011), No. 1, pp. 79-83

DOI 10.4134/BKMS.2011.48.1.079

\title{
THE LOWER AUTOCENTRAL SERIES OF ABELIAN GROUPS
}

\author{
Mohammad Reza R. Moghaddam, Foroud Parvaneh, \\ and Mohammad Naghshineh
}

\begin{abstract}
In the present paper we introduce the lower autocentral series of autocommutator subgroups of a given group. Following our previous work on the subject in 2009, it is shown that every finite abelian group is isomorphic with $n^{t h}$-term of the lower autocentral series of some finite abelian group.
\end{abstract}

\section{Introduction}

Let $A=\operatorname{Aut}(G)$ denote the group of automorphisms of a given group $G$. For any element $g \in G$ and $\alpha \in A$ the element $[g, \alpha]=g^{-1} g^{\alpha}$ is an autocommutator of $g$ and $\alpha$. We define the autocommutator of higher weight inductively as follows:

$$
\left[g, \alpha_{1}, \alpha_{2}, \ldots, \alpha_{i}\right]=\left[\left[g, \alpha_{1}, \alpha_{2}, \ldots, \alpha_{i-1}\right], \alpha_{i}\right]
$$

for all $\alpha_{1}, \alpha_{2}, \ldots, \alpha_{i} \in A$.

So the autocommutator subgroup of weight $i+1$ is defined in the following way:

$$
K_{i}(G)=[G, \underbrace{A, \ldots, A}_{i \text {-times }}]=\left\langle\left[g, \alpha_{1}, \alpha_{2}, \ldots, \alpha_{i}\right] \mid g \in G, \alpha_{1}, \alpha_{2}, \ldots, \alpha_{i} \in A\right\rangle .
$$

Clearly $K_{i}(G)$ is a characteristic subgroup of $G$ for all $i \geq 1$. Therefore, one obtains a descending chain of autocommutator subgroups of $G$ as follows:

$$
G \supseteq K_{1}(G) \supseteq K_{2}(G) \supseteq \cdots \supseteq K_{i}(G) \supseteq \cdots,
$$

which we may call it the lower autocentral series of $G$. The aim of the present paper is to prove the following main result.

Theorem 1.1. For any finite abelian group $G$ and every natural number $n \in \mathbb{N}$, there exists a finite abelian group $H$ such that

$$
G \cong K_{n}(H) \text {. }
$$

Received May 11, 2009.

2010 Mathematics Subject Classification. 20D45, 20D25, $20 \mathrm{E} 34$.

Key words and phrases. autocommutator subgroup, autocentral series, abelian group. 


\section{Preliminary results}

In order to prove our main result, we need to prove some technical lemmas, which are interested in their own right.

Lemma 2.1. Let $G=H \times K$ be the direct product of two characteristic subgroups $H$ and $K$. Then for any natural number $n$,

$$
K_{n}(H \times K)=K_{n}(H) \times K_{n}(K) .
$$

Proof. Clearly every element $g \in G$ can be written as $g=h k$, where $h \in H$ and $k \in K$. By Lemma 2.1 of [5],

$$
\operatorname{Aut}(G) \cong \operatorname{Aut}(H) \times \operatorname{Aut}(K) .
$$

Using induction on $n$, we show that

$$
\left[h k, \alpha_{1}, \alpha_{2}, \ldots, \alpha_{n}\right]=\left[h, \alpha_{1 \mid H}, \alpha_{2 \mid H}, \ldots, \alpha_{n \mid H}\right]\left[k, \alpha_{1 \mid K}, \alpha_{2 \mid K}, \ldots, \alpha_{n \mid K}\right],
$$

for all $\alpha_{1}, \alpha_{2}, \ldots, \alpha_{n} \in \operatorname{Aut}(G)$. If $n=1$, then since the subgroups $H$ and $K$ are characteristic in $G$ and $h k=k h$, we have

$$
\left[h k, \alpha_{1}\right]=\left[h, \alpha_{1}\right]\left[k, \alpha_{1}\right] .
$$

Now, assume the result holds for $n-1$, then

$$
\begin{aligned}
& {\left[h k, \alpha_{1}, \alpha_{2}, \ldots, \alpha_{n}\right] } \\
= & {\left[\left[h k, \alpha_{1}, \alpha_{2}, \ldots, \alpha_{n-1}\right], \alpha_{n}\right] } \\
= & {\left[\left[h, \alpha_{1 \mid H}, \alpha_{2 \mid H}, \ldots, \alpha_{n-1 \mid H}\right]\left[k, \alpha_{1 \mid K}, \alpha_{2 \mid K}, \ldots, \alpha_{n-1 \mid K}\right], \alpha_{n}\right] } \\
= & {\left[h, \alpha_{1 \mid H}, \alpha_{2 \mid H}, \ldots, \alpha_{n-1 \mid H}, \alpha_{n \mid H}\right]\left[k, \alpha_{1 \mid K}, \alpha_{2 \mid K}, \ldots, \alpha_{n-1 \mid K}, \alpha_{n \mid K}\right] . }
\end{aligned}
$$

This implies that $K_{n}(G) \subseteq K_{n}(H) \times K_{n}(K)$.

By Lemma 2.1 of [5], any automorphism $\mu$ of $H$, can be extended to an automorphism $\bar{\mu}$ of $G$. Hence, for all $\mu_{1}, \ldots, \mu_{n} \in \operatorname{Aut}(H)$ and $h \in H$

$$
\left[h, \mu_{1}, \ldots, \mu_{n}\right]=\left[h, \overline{\mu_{1}}, \ldots, \overline{\mu_{n}}\right] \in K_{n}(G) .
$$

Therefore $K_{n}(H) \subseteq K_{n}(G)$. Similarly, $K_{n}(K)$ is contained in $K_{n}(G)$. Thus

$$
K_{n}(G)=K_{n}(H) \times K_{n}(K) .
$$

Using the above notation, we have the following

Lemma 2.2. If $G$ is a finite cyclic group, then for any natural number $n$,

$$
K_{n}(G)=G^{2^{n}} \text {. }
$$

Proof. Let $G=\left\langle x \mid x^{m}=1\right\rangle$ be the cyclic group of order $m$. Clearly $\varphi: x \mapsto x^{i}$ is an automorphism of $G$ if and only if $(i, m)=1$. Since $G$ is abelian, it follows that $\alpha: x \mapsto x^{-1}$ is an automorphism. So by an easy induction, for all $g \in G$, if $n$ is even

$$
g^{2^{n}}=[g, \underbrace{\alpha, \ldots, \alpha}_{n \text {-times }}] \in G^{2^{n}},
$$


and if $n$ is odd, then

$$
g^{2^{n}}=[g^{-1}, \underbrace{\alpha, \ldots, \alpha}_{n \text {-times }}] \in G^{2^{n}},
$$

which implies that $G^{2^{n}}$ is contained in $K_{n}(G)$.

Conversely, assume $\left(2^{n}, m\right)=1$ then $m$ is odd number and hence $G=G^{2^{n}}$, which follows that $K_{n}(G) \subseteq G^{2^{n}}$. Now, assume $\left(2^{n}, m\right) \neq 1$, i.e., $m$ is an even number then using an easy induction for all $\alpha_{1}, \alpha_{2}, \ldots, \alpha_{n} \in \operatorname{Aut}(G)$, we obtain that

$$
\left[g, \alpha_{1}, \alpha_{2}, \ldots, \alpha_{n}\right]=g^{\left(i_{1}-1\right) \cdots\left(i_{n}-1\right)} \in G^{2^{n}},
$$

where $\alpha_{j}(g)=g^{i_{j}},\left(i_{j}, m\right)=1$ that is to say $i_{j}-1$ is even, for all $1 \leq j \leq n$. This completes the proof.

Lemma 2.3. Let $G$ be a finite abelian group of odd order $m$ and $Z_{2}$ the cyclic group of order 2. Then $K_{n}(G)$ and $K_{n}\left(G \times Z_{2}\right)$ are both isomorphic with $G$ for all natural number $n$.

Proof. Clearly, by the assumption $\left(2^{n}, m\right)=1$ and hence by Lemma 2.2 , $K_{n}(G)=G$.

One notes that $G$ and $Z_{2}$ are both characteristic subgroups in the direct product $G \times Z_{2}$. Therefore, Lemma 2.1 implies that

$$
K_{n}\left(G \times Z_{2}\right)=K_{n}(G) \times K_{n}\left(Z_{2}\right) .
$$

Now, the triviality of $K_{n}\left(Z_{2}\right)$ gives the result.

The following proposition is very useful in our further investigations.

Proposition 2.4. Let $G$ be a cyclic group of order $2^{m}$ and $H$ be an abelian 2-group of exponent $2^{n}$ with $n<m$. Then

$$
K_{n}(G \times H)=G^{2^{n}} \times H^{2^{n-1}} .
$$

Proof. Let $G=\left\langle g \mid g^{2^{m}}=1\right\rangle$ be the cyclic group of order $2^{m}$. Then we define the automorphisms $\alpha_{h}$ and $\alpha_{h}^{\prime}$ of the group $G \times H$, given by $g^{\alpha_{h}}=g h, h^{\alpha_{h}}=h$ and $g^{\alpha_{h}^{\prime}}=g h^{-1}, h^{\alpha_{h}^{\prime}}=h^{-1}$ for all $h \in H$.

Now, if $n$ is even, then

$$
h^{2^{n-1}}=[g, \underbrace{\alpha_{h}^{\prime}, \ldots, \alpha_{h}^{\prime}}_{n \text {-times }}],
$$

and if $n$ is odd we have

$$
h^{2^{n-1}}=[g, \alpha_{h}, \underbrace{\alpha_{h}^{\prime}, \ldots, \alpha_{h}^{\prime}}_{(n-1) \text {-times }}] .
$$

These imply that $H^{2^{n-1}} \subseteq K_{n}(G \times H)$ and $G^{2^{n}} \subseteq K_{n}(G) \subseteq K_{n}(G \times H)$. Thus

$$
G^{2^{n}} \times H^{2^{n-1}} \subseteq K_{n}(G \times H) \text {. }
$$


On the other hand, using the structures of the groups $G$ and $H$ for all $\alpha_{1}, \alpha_{2}, \ldots, \alpha_{n} \in \operatorname{Aut}(G \times H)$ and $x \in G \times H$,

$$
\left[x, \alpha_{1}, \alpha_{2}, \ldots, \alpha_{n}\right] \in G^{2^{n}} \times H^{2^{n-1}},
$$

which implies that $K_{n}(G \times H) \subseteq G^{2^{n}} \times H^{2^{n-1}}$ and the proof is complete.

The following theorem follows from the above proposition, which is interested in its own right.

Theorem 2.5. For all natural numbers $m \geq n_{1} \geq \cdots \geq n_{r}$ and $n \geq 2$,

$$
K_{n}\left(Z_{2^{m}} \times Z_{2^{n_{1}}} \times \cdots \times Z_{2^{n_{r}}}\right)=Z_{2^{m-n}} \times Z_{2^{n_{1}-(n-1)}} \times \cdots \times Z_{2^{n_{r}-(n-1)}} .
$$

Proof of Theorem 1.1. Let $G$ be a finite abelian group, which can be written as a product of its Sylow subgroups. Now, if $|G|$ is an odd number, then by Lemma 2.3,

$$
G=K_{n}(G) .
$$

Assume 2 divides $|G|$ and $A$ is the Sylow 2-subgroup of $G$, then $G=A \times P_{1} \times$ $\cdots \times P_{s}$, where $P_{i}^{\prime} s$ are Sylow $p_{i}$-subgroups of $G(1 \leq i \leq r)$. By Lemma 2.1,

$$
K_{n}(G)=K_{n}(A) \times P_{1} \times \cdots \times P_{s} .
$$

As $A$ is an abelian 2-group, we may write $A$ as a direct product of cyclic groups of orders some powers of 2 , as follows:

$$
A \cong Z_{2^{m}} \times Z_{2^{n_{1}}} \times \cdots \times Z_{2^{n_{r}}},
$$

where $m \geq n_{1} \geq \cdots \geq n_{r}$.

Now, we choose the abelian group

$$
H=Z_{2^{m+n}} \times Z_{2^{n_{1}+n-1}} \times \cdots \times Z_{2^{n_{r}+(n-1)}} \times P_{1} \times \cdots \times P_{s} .
$$

It can be easily seen that

$$
K_{n}(H)=G,
$$

and hence the claim is proved.

\section{References}

[1] C. Chiš, M. Chiš, and G. Silberberg, Abelian groups as autocommutator groups, Arch. Math. (Basel) 90 (2008), no. 6, 490-492.

[2] M. Deaconescu and G. L. Walls, Cyclic groups as autocommutator groups, Comm. Algebra 35 (2007), no. 1, 215-219.

[3] P. Hegarty, The absolute centre of a group, J. Algebra 169 (1994), no. 3, 929-935.

[4] - Autocommutator subgroups of finite groups, J. Algebra 190 (1997), no. 2, 556562.

[5] M. Naghshineh, M. R. R. Moghaddam, and F. Parvaneh, The third term of the lower autocentral series of abelian groups, Journal of Mathematical Extension, Vol. 4, No. 1 (2009), 1-6.

[6] D. J. S. Robinson, A Course in the Theory of Groups, Graduate Texts in Mathematics, 80. Springer-Verlag, New York-Berlin, 1982. 
Mohammad Reza R. Moghaddam

Faculty of Mathematical Sciences

FERDoWsi UNIVERSity OF MASHHAD

AND

Centre of Excellence in Analysis on Algebraic Structures

MASHHAD, IRAN

E-mail address: rezam@ferdowsi.um.ac.ir

Foroud Parvaneh

Department of Mathematics

ISLAMIC AZAD UNIVERSITY

MashHad-Branch, MashHad, Iran

E-mail address: fparvaneh@iauksh.ac.ir

MOHAmmad Naghshineh

Department of Mathematics

ISLAMIC AZAD UNIVERSITY

MashHad-Branch, MashHad, Iran

E-mail address: mnaghinehfard@yahoo.com 